\title{
EL LEGADO DEL "SEXENIO DE LA CORRUPCIÓN”: LOS RETOS DEL SISTEMA NACIONAL ANTICORRUPCIÓN
}

\section{THE LEGACY OF THE "SEXENNIUM OF CORRUPTION": THE CHALLENGES OF THE NATIONAL ANTI-CORRUPTION SYSTEM}

\author{
L'HÉRITAGE DU «GOUVERNEMENT \\ DE LA CORRUPTION»: LES DÉFIS POSÉS \\ AU SYSTÈME NATIONAL ANTICORRUPTION
}

\author{
Fernando Nieto Morales \\ El Colegio de México \\ fnieto@colmex.mx
}

Resumen: Este artículo analiza el desempeño del gobierno de Enrique Peña Nieto en materia de combate a la corrupción. Me enfoco en entender los orígenes del Sistema Nacional Anticorrupción (sNA), cómo está formado, y en identificar los retos y posibilidades de éxito de cara al futuro. Con base en la revisión de la evidencia reunida, propongo que, en materia de anticorrupción, el desempeño del gobierno de Peña Nieto fue reactivo, incompleto y limitado. Los esfuerzos respondieron principalmente a la necesidad de aplacar el descontento social, mientras que los recursos se mantuvieron precarios y nunca se formuló una política anticorrupción eficaz. Más aún, no hubo voluntad política para consolidar y mejorar la estrategia en ciernes, ni una coalición que asegurara su continuidad. Estos defectos explican por qué el sNa no ha podido consolidarse, hasta ahora, como una estrategia anticorrupción de largo plazo.

Palabras clave: Sistema Nacional Anticorrupción (SNA); corrupción; plataforma digital nacional; Enrique Peña Nieto; reforma constitucional.

Abstract: This paper analyses the performance of Enrique Peña Nieto's government with regard to the fight against corruption. It focuses on understanding the origins of the National Anti-Corruption System (SNA), how it is structured, and identifying the challenges and possibilities of success in the future. Based on a review of the evidence, it proposes that with regard to cor- 
ruption the performance of the Peña Nieto government was reactive, incomplete, and limited. The efforts made responded principally to the need to placate social discontent, while remaining under-resourced and lacking in an effective anti-corruption policy. Furthermore, there was no political will to consolidate and improve the emerging strategy, or a coalition to ensure its continuity. These defects explain why the SNA has failed to consolidate itself, to date, as a long-term anti-corruption strategy.

Keywords: National Anti-Corruption System (SNA); corruption; national digital platform; Enrique Peña Nieto; constitutional reform.

\section{Traducción de Gonzalo Celorio Morayta}

RÉSumÉ: Cet article examine les résultats qu'a obtenus le gouvernement d'Enrique Peña Nieto dans sa lutte contre la corruption. On s'interroge sur les origines du Système national anticorruption (SNA), sa structure, les défis auxquels il doit faire face et ses possibilités de succès dans l'avenir. L'évidence disponible permet de dire que les mesures de ce gouvernement ont été réactives et insuffisantes. Limités par le manque de ressources et par l'incapacité à formuler une politique efficace contre la corruption, les efforts déployés ont essayé surtout d'apaiser le mécontentement social. En outre, il n'y a pas eu une volonté politique de consolider et d'améliorer la stratégie naissante, ni de former une coalition pour lui assurer la continuité. Toutes ces défaillances ont empêché le SNA de consolider une politique de long terme contre la corruption.

Mots clefs: Système national anticorruption (SNA); corruption; plateforme numérique nationale; Enrique Peña Nieto; réforme constitutionnelle.

Traducción de Bernardo Mabire

Fecha de recepción: junio de 2019

Fecha de aceptación: noviembre de 2019 


\section{INTRODUCCIÓN}

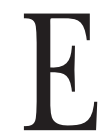

n materia de combate a la corrupción, el sexenio de Enrique Peña Nieto comenzó con muchas expectativas. Empero, a pesar de promesas y discursos, el sexenio 2012-2018 estuvo plagado de escándalos de corrupción -de hecho, algunos muy cercanos al presidente, su familia y a su grupo político, el "nuevo PRI"-. Pero, sobre todo, fue un sexenio en el que, a juzgar por los indicadores de percepción pública, la corrupción en la política y en la administración pública no sólo no menguó, sino que acaso se hizo más evidente.

Según datos de la Encuesta Nacional de Calidad e Impacto Gubernamental, la corrupción fue el segundo problema que más preocupó a los mexicanos en 2015 y de nuevo en 2017, sólo por debajo de la inseguridad. ${ }^{1}$ En 2017, el país cayó cinco lugares en el Índice de Percepciones de la Corrupción de Transparencia Internacional y otros tres un año después. ${ }^{2}$ Esto lo colocó por debajo de todos sus competidores regionales y muy por debajo de los líderes globales. En el mismo periodo, México tuvo el segundo peor desempeño de los países miembros de la Organización para la Cooperación y el Desarrollo Económicos (OCDE) en casi todos los indicadores de buena gobernanza y control de la corrupción del Banco Mundial, ${ }^{3}$ así como en el índice de Estado de derecho del World Justice Project. ${ }^{4}$

No obstante, y quizá paradójicamente, el sexenio de Peña Nieto también vio nacer una nueva estrategia de combate a la

${ }^{1}$ Instituto Nacional de Estadística y Geografía, INEGi, Encuesta Nacional de Calidad e Impacto Gubernamental (ENCIG) 2017, www.beta. inegi.org.mx/programas/encig

2 Transparency International, Corruption Perceptions Index, www. transparency.org

${ }^{3}$ World Bank, Worldwide Governance Indicators, https://info.worldbank.org/governance/wgi

${ }^{4}$ World Justice Project, The World Justice Project: rule of law index 2017 2018, Washington, The World Justice Project, 2018, pp. 6-7. 
corrupción y la impunidad. En 2015, el Sistema Nacional Anticorrupción (SNA) surgió como la apuesta más reciente -y acaso la más ambiciosa hasta ahora- para atajar el problema. Este sistema fue el resultado de una reforma constitucional que dio lugar a nuevos tipos penales y administrativos, creó un nuevo mecanismo de coordinación entre autoridades federales, estatales y municipales, y abrió la puerta a la participación ciudadana en la formulación de la política nacional y de las políticas estatales anticorrupción. Según el modelo de John W. Kingdon, las ventanas para la adopción de reformas dependen de la confluencia de tres conjuntos de factores: la identificación de un problema público, la existencia de soluciones (o precedentes) y un clima político propicio. ${ }^{5}$ Como lo explico más adelante, la combinación de un entorno político adverso a la administración peñista, el señalamiento de un problema de corrupción e impunidad extendidas, la participación y presión de un grupo de activistas y académicos, y el precedente de reformas con soluciones organizacionales similares resultó en la formulación del nuevo sistema.

A pesar de que la reforma fue aplaudida por tirios y troyanos, tras el fin del sexenio peñista es claro que el sNA enfrenta grandes retos de implementación. ${ }^{6}$ Éstos incluyen importantes problemas de diseño, recursos menguantes, relaciones intergubernamentales complejas y la falta de cooperación de los receptores y operadores de la reforma. Quizá este conjunto de retos explica la incredulidad de muchos: según una encuesta de 2016, 54\% de una muestra representativa nacional opinaba que el SNA ayudaría poco o nada a reducir la corrupción en el país. ${ }^{7}$ En todo caso, los problemas de implementa-

${ }^{5}$ John W. Kingdon, Agendas, alternatives and public policies, Pearson, Crawfordsville, 2011.

6 Véase Michael Hill y Peter Hupe, Implementing public policy, Londres, Sage, 2002, pp.120-136.

${ }^{7}$ Parametría, Carta paramétrica, "Más mexicanos desconocen el Sistema Nacional Anticorrpución”, www.parametria.com.mx/carta_parame trica.php? cp=4897 (consulta del 14 de diciembre de 2018). 
ción, la debilidad institucional y operativa del SNA y la victoria de López Obrador en la elección de 2018 -quien ha criticado el sistema por ser "un gasto improductivo e innecesario"-8 pintan un panorama poco promisorio.

Mi principal argumento es que la estrategia anticorrupción de Peña Nieto fue, haciendo un balance, reactiva, incompleta y limitada. Fue reactiva en tanto respondió principalmente a la necesidad de aplacar el descontento social ante los escándalos de corrupción e impunidad. Fue incompleta porque los recursos se mantuvieron precarios y nunca se formuló una política anticorrupción coherente (o al menos no en los primeros años de implementación). Y fue limitada porque no hubo voluntad política para consolidar y mejorar el sistema, ni una coalición que asegurara decisivamente su continuidad. Estos defectos de origen derivaron en la configuración del sNA y, en particular, en los problemas que ha venido enfrentando en su adopción e implementación. A menos de que se logre articular una coalición anticorrupción suficientemente poderosa y duradera, es poco probable que el SNA se convierta en una estrategia efectiva y de largo aliento.

¿Cómo se relacionan los escándalos y la respuesta del gobierno de Enrique Peña Nieto con el surgimiento del SNA? ¿Cómo se formó? ¿Cuáles son los retos y las posibilidades de éxito del nuevo sistema? Este texto pretende dar respuesta a estas preguntas. Mi objetivo, pues, es presentar una lectura de algunos de los principales sucesos y considerar los claroscuros en materia de política de combate a la corrupción durante el sexenio peñista, así como señalar las principales debilidades y los retos del SNA de cara al futuro inmediato.

${ }^{8}$ Carina García, "López Obrador anticipa fracaso del Sistema Anticorrupción”, El Universal, 3 de octubre de 2016. 
Antecedentes

$\mathrm{Al}$ menos desde los años ochenta, ha habido varios esfuerzos de construcción institucional y de formulación de estrategias de combate y prevención de la corrupción. ${ }^{9}$ Se han creado burocracias (como la Secretaría de la Función Pública, SFP, y la Auditoría Superior de la Federación); ha habido importantes reformas legales (por ej., la promulgación de la Ley Federal de Responsabilidades de los Servidores Públicos de 1982 o la Ley Federal de Transparencia y Acceso a la Información Pública de 2002); y se han diseñado programas administrativos de combate a la corrupción, transparencia y mejora de la gestión en el ámbito federal (por ej., el Programa Nacional de Combate a la Corrupción y Fomento a la Transparencia y el Desarrollo Administrativo 2001-2006 o el Programa Especial de Mejora de la Gestión 2008-2012). Como dice Mauricio Dussauge: "La amplitud y diversidad de los cambios y estrategias implementados parecieran ser [...] reflejo tanto de la tenacidad y persistencia del problema como de su complejidad intrínseca". ${ }^{10}$ Aunque estos esfuerzos no siempre fueron eficientes, sí sentaron bases y han arrojado lecciones para avanzar en la construcción de nuevas instituciones y mejorar el diseño de las políticas anticorrupción. ${ }^{11}$

${ }^{9}$ David Arellano Gault y Jesús F. Hernández Galicia, “¿Control interno o lucha contra la corrupción? Hacia un verdadero control interno en la administración pública federal mexicana”, en María del Carmen Pardo y Guillermo M. Cejudo (eds.), Trayectorias de reformas administrativas en México: legados y conexiones, México, El Colegio de México, 2016, pp.149184; Guillermo Cejudo, "Explaining change in the Mexican public sector: the limits of New Public Management", International Review of Administrative Sciences, vol. 74, núm. 1 (2008), pp. 111-127.

${ }^{10}$ Mauricio Dussauge, "Combate a la corrupción y rendición de cuentas: avances, limitaciones, pendientes y retrocesos”, en José Luis Méndez (coord.), Los grandes problemas de México: XIII Políticas Públicas, México, El Colegio de México, 2010, pp.244-245.

${ }^{11}$ Véanse las contribuciones en el volumen editado por Guillermo M. Cejudo, Sergio López Ayllón y Alejandra Ríos Cázares, La política de transparencia en México. Instituciones, logros y desafios, México, CIDE, 2012. 
En la campaña presidencial de 2012 y siguiendo una fórmula que aparentemente había resultado provechosa como candidato a gobernador del Estado de México, Peña Nieto firmó más de 260 compromisos ante notario público, incluyendo varios en materia de transparencia, rendición de cuentas y combate a la corrupción. ${ }^{12}$ Entre éstos, se propuso la creación de un nuevo órgano anticorrupción que substituiría a la Secretaría de la Función Pública -mucho más identificada con los programas de modernización administrativa y de combate a la corrupción de los gobiernos panistas.

Tras la elección de 2012 y en el marco del Pacto por México, ${ }^{13}$ se presentaron dos iniciativas. Primero, en septiembre de 2012, el presidente electo envió una iniciativa de reforma constitucional en materia de transparencia por conducto de la fracción parlamentaria de su partido en el Senado de la República. Ésta ampliaba considerablemente las facultades del entonces Instituto Federal de Acceso a la Información Pública (IFAI), dotándolo de autonomía constitucional y de atribuciones para revisar la garantía del derecho de acceso a la información en los tres ámbitos de gobierno y en los órganos autónomos del Estado.

La segunda iniciativa, presentada por el senador Emilio Gamboa a nombre de los grupos parlamentarios del PRI y del Partido Verde Ecologista, contenía varias reformas a la Cons-

12 "Los compromisos que Peña Nieto tendrá que cumplir", Animal Político, 2 de julio de 2012.

${ }^{13}$ Como parte del Pacto por México, se establecieron tres acuerdos en materia de transparencia, rendición de cuentas y combate a la corrupción: 1) implementar una reforma para la rendición de cuentas contables, 2) ampliar las facultades del Instituto Federal de Transparencia y Acceso a la Información, dotarlo de autonomía y promover que los órganos estatales de acceso a la información sean autónomos y colegiados, y 3) establecer una comisión nacional y comisiones estatales anticorrupción con facultades de prevención, investigación, sanción administrativa y denuncia, así como crear un "Consejo Nacional para la Ética Pública" con la participación de autoridades, academia y sociedad civil (véase Alfonso Myers Gallardo y Diana L. Rouzand Anaya, "El Sistema Nacional Anticorrupción”, Estudios, vol. 14, núm. 119 (2016), pp. 135-159). 
titución en materia de combate a la corrupción. La minuta proponía la creación de una Comisión Nacional Anticorrupción con capacidad de prevención, investigación y sanción de actos de corrupción, así como la creación de un Consejo Nacional por la Etica Pública. ${ }^{14}$ Según la iniciativa, la Comisión sería un órgano colegiado con autonomía presupuestal y de gestión, y tendría a su cargo, entre otras facultades, investigar actos de corrupción en todos los ámbitos de gobierno, impulsar programas de prevención y formular recomendaciones y acciones para promover la ética y la honestidad en el servicio público.

La propuesta de crear una comisión nacional respondía a una tendencia global a favor de crear cuerpos especializados en el combate y la prevención de la corrupción, pero la propuesta también se inscribía en una larga trayectoria de esfuerzos del Estado mexicano por hacer frente a la corrupción endémica. ${ }^{15}$ Como ya lo ha apuntado Miguel Ángel Valverde, tanto la creación de una nueva burocracia como de leyes que la dotan de capacidades de investigación y sanción son dos tendencias que caracterizan la mayoría de los esfuerzos de construcción institucional en materia anticorrupción en México. ${ }^{16}$ A pesar de que muchos detalles nunca fueron del todo aclarados, la propuesta de crear una Comisión Nacional Anticorrupción, en principio, sí implicaba cambios importantes respecto a las estrategias de gobiernos anteriores. Por ejemplo, significaba extraer la política anticorrupción de la

14 "Iniciativa con proyecto de decreto que reforma y adiciona los artículos 22, 73, 79, 105, 107, 109, 113, 116 y 122 de la Constitución Política de los Estados Unidos Mexicanos que presentan las senadoras y los senadores integrantes del grupo parlamentario del Partido Revolucionario Institucional y del Partido Verde Ecologista de México del Senado de la República".

15 Véase Dussauge, art. cit.

16 Miguel Ángel Valverde, "Mecanismos institucionales contra la corrupción”, en Fernando Nieto y Ernesto Velasco (eds.), Una agenda para la administración pública. Reconocimiento a la trayectoria de María del Carmen Pardo, México, El Colegio de México, 2018, pp. 265-296. 
Administración Pública Federal y desaparecer la sfr. ${ }^{17}$ No obstante, la propuesta no prosperó en la Cámara de Diputados y la creación de la comisión nunca se concretó.

En cambio, la reforma en materia de transparencia sí fue exitosa. Tras un proceso de dos años, en febrero de 2014, el Congreso promulgó una reforma constitucional que creó al Instituto Nacional de Transparencia, Acceso a la Información y Protección de Datos Personales (INAI) en substitución del IFAI. ${ }^{18}$ Los cambios incluían una nueva estructura de gobierno del instituto (por ej., se pasaba de 5 a 7 comisionados nombrados por el Senado de la República y se creó un Consejo Consultivo integrado por diez ciudadanos independientes, también seleccionados por el Senado), así como un nuevo marco normativo. Éste incluyó la Ley General de Transparencia y Acceso a la Información (promulgada en 2015), la capacidad de promover acciones de inconstitucionalidad desde el INAI y, quizá más importante, un aumento muy notable en el número de sujetos obligados (los tres poderes y órganos autónomos en los tres niveles de gobierno, partidos políticos, sindicatos, fideicomisos y fondos públicos y todas las personas físicas o morales que reciben recursos públicos). A su vez, la Ley General de Transparencia creó el Sistema Nacional de Transparencia, Acceso a la Información Pública y Protección de Datos Personales (sNT). Éste integra al INAI todos los órganos garantes del país, a saber, la Auditoría Superior de la Federación, el Archivo General de la Nación y el Instituto Nacional de Estadística y Geografía. El sNT tiene por objeto

${ }^{17}$ Aunque la propuesta de desaparecer a la SFP ya se había planteado durante la administración de Felipe Calderón en 2009 y durante los primeros años del gobierno de Peña Nieto, la dependencia se mantuvo sin titular y en proceso de extición desde 2013 (véase "Decreto por el que se reforman, adicionan y derogan diversas disposiciones de la Ley Orgánica de la Administración Pública Federal”, Diario Oficial de la Federación, 2 de enero de 2013).

18 "Decreto por el que se reforman y adicionan diversas disposiciones de la Constitución Política de los Estados Unidos Mexicanos, en materia de transparencia”, Diario Oficial de la Federación, 7 de febrero de 2014. 
coordinar acciones conjuntas para la formulación de una política pública transversal (nacional) en materia de transparencia y acceso a la información. ${ }^{19}$

Las características de la reforma en materia de transparencia son interesantes porque, como veremos, representan un antecedente normativo y organizacional para la reforma constitucional que finalmente dio origen al Sistema Nacional Anticorrupción. La forma en que se negoció la reforma de 2014 también es relevante. En particular, durante su discusión en el Senado se promovió la participación activa de expertos, académicos y organizaciones de la sociedad civil. ${ }^{20}$ De hecho, varias de sus características en materia de transparencia respondieron directamente a recomendaciones técnicas y opiniones que se expresaron en los foros de la Comisión de Puntos Constitucionales. ${ }^{21}$ Como ejercicio de parlamento abierto, la reforma en materia de transparencia de 2014 sentó precedente al abrir la puerta a expertos y organizaciones civiles, y permitirles influir decisivamente en el proceso legislativo.

\section{ESFUERZOS ANTICORRUPCIÓN DURANTE EL PEÑISMO:}

\section{LA REFORMA DE 2015}

El último cuatrimestre de 2014 fue un parteaguas para la administración de Peña Nieto. En septiembre de ese año, la noticia de la desaparición forzada de 43 estudiantes en el estado de Guerrero movilizó a cientos de miles en todo el país en protestas contra el gobierno. El 9 de noviembre del mismo año, el reportaje que dio a conocer la "Casa Blanca"

${ }^{19}$ Ley Gral. de Transparencia y Acceso a la Información, 2015, arts.2731; también, Sistema Nacional de Transparencia, www.snt.org.mx

${ }^{20}$ Alejandra Lajous (coord.), La sociedad civilvs. la corrupción, México, Grijalbo, 2019.

${ }^{21}$ Guillermo Ávila, "Citizen participation in legislative power: An argument to strengthen representation”, en Eduardo Bohórquez (coord.), Open parliaments: Results and expectations, México, Transparencia Mexicana, 2015, pp. 57-70. 
de la esposa del presidente generó nuevas manifestaciones de rechazo y avivó el descontento. ${ }^{22}$ Ambos sucesos marcaron el fin del primer periodo de reformas dominado por el Pacto por México y señalaron el comienzo de la crisis del peñismo como proyecto político. ${ }^{23}$ Como apunta Mauricio Merino, "en menos de 45 días, el desencanto social acumulado por casi tres lustros, concentró la indignación colectiva en [...] dos causas: la impunidad y la corrupción". 24

En lo que toca a la Casa Blanca, la reacción inmediata de la administración fue remedial. El mismo día del reportaje, la Oficina de la Presidencia aclaró que la casa pertenecía a Angélica Rivera, esposa de Peña Nieto, pero no desconoció la conexión con Grupo Higa -que, en ese momento, participaba en la licitación para la construcción de un tren de alta velocidad entre Ciudad de México y Querétaro-. Unos días después, el 18 de noviembre, por medio de un mensaje televisado, Rivera declaró que la casa en efecto era suya y que sus ingresos como actriz le habían permitido comprarla. ${ }^{25} \mathrm{La}$ respuesta fue insuficiente. Más aún, tuvieron que pasar casi dos meses para que el gobierno federal iniciara una investigación oficial. Misma que, en agosto de 2015, exoneró al pre-

22 En muy apretada síntesis, el caso refiere al descubrimiento, a raíz de un reportaje periodístico, de una mansión que la esposa del presidente había adquirido de una filial del Grupo Higa en una de las zonas de mayor plusvalía de la Ciudad de México y con valor aproximado de 7 millones de dólares. Grupo Higa fue una de las empresas más favorecidas con contratos públicos durante el gobierno de Peña Nieto cuando fue gobernador del Estado de México y una de las constructoras involucradas en megaproyectos de infraestructura del gobierno de la República después de 2012.

${ }^{23}$ Para una revisión de las reformas impulsadas durante del primer año de la administración de Peña Nieto, véase Fernando Barrientos y Daniel Añorve, "México 2013: Acuerdos, reformas y descontento", Revista de Ciencia Política, vol. 34, núm. 1 (2014), pp.221-247.

${ }^{24}$ Mauricio Merino, México: La batalla contra la corrupción, Washington D.C., Wilson Center, 2015, p. 2.

${ }^{25}$ Jessica Corona, "Las reacciones del presidente a lo ocurrido en su tercer año”, Milenio, 1 de septiembre de 2015. 
sidente y a su esposa. Un resultado que, de nuevo, resultó insatisfactorio para la opinión pública y la oposición. ${ }^{26}$

En ese contexto se negoció la reforma constitucional en materia anticorrupción de 2015 en el Congreso de la Unión. Es posible que la conjunción de eventos, ${ }^{27}$ así como el agotamiento del Pacto por México, debilitaran seriamente la posición del gobierno peñista y contribuyeran a crear una ventana de oportunidad para la reforma.

\section{CONDICIONES PARA LA ADOPCIÓN DE LA REFORMA}

Como ya mencioné antes, la propuesta original de Peña Nieto de crear una Comisión Nacional Anticorrupción fue infructuosa. De hecho, los esfuerzos de construcción institucional en materia de combate a la corrupción quedaron estancados desde 2013. ${ }^{28}$ Sin embargo, hacia principios de 2015 hubo un renovado interés en el Congreso por retomar los esfuerzos de reforma. Este impulso se explica por la conjunción de al menos tres grupos de factores. ${ }^{29}$

Primero, el ánimo político tras 2014 y, en particular, tras los escándalos y sospechas de corrupción y de conflicto de intereses en torno a la administración federal y de miembros prominentes del nuevo PRI (y otros partidos) terminaron por

${ }^{26}$ Véase, por ej., Sandra Rodríguez Nieto et al., "La explicación de la SFP sobre las casas no convence ni a los viejos amigos de Peña Nieto: PRD y PAN", Sin Embargo, 23 de agosto de 2015.

${ }^{27}$ En el mismo periodo también se conoció de la fuga de Joaquín "El Chapo" Guzmán; se hizo público el reportaje de los abusos de militares en el pueblo de Tlatlaya, Estado de México; y el peso mexicano se depreció considerablemente.

${ }^{28}$ Una excepción fue la creación, mediante acuerdo, de una Fiscalía especializada en materia de delitos relacionados con hechos de corrupción dentro de la Fiscalía General de la República ("Acuerdo A/011/14 por el que se crea la Fiscalía Especializada en Materia de Delitos Relacionados con Hechos de Corrupción y se establecen sus atribuciones", Diario Oficial de la Federación, 12 de marzo de 2014).

${ }^{29}$ Véase Kingdon, op. cit. 
crear condiciones que pusieron presión sobre los actores políticos. En particular, la administración peñista y sus aliados en el Congreso se vieron en la necesidad de reaccionar ante el creciente descontento. En febrero de 2015, tras casi dos años sin titular, Peña Nieto detuvo la extinción de la SFP y nombró un secretario. El mandato explícito del nuevo secretario, Virgilio Andrade, fue investigar el caso de la "Casa Blanca”, así como de proponer (nuevas) medidas anticorrupción. Éstas incluyeron, por ejemplo, la creación de la Unidad Especializada de Ética y de Prevención de Conflictos de Intereses, así como ajustes en los procesos de verificación patrimonial. No obstante, no parece que esas medidas reactivas $-y$ de carácter eminentemente administrativo- fueran suficientes. ${ }^{30}$ La reacción tardía de la administración atizó la protesta y dio oportunidad a otros actores de participar en la agenda.

Por un lado, organizaciones de la sociedad civil, como Transparencia Mexicana y el Instituto Mexicano para la Competitividad, ya habían impulsado la campaña “\#3de3” en la que denunciaban los escándalos de corrupción e invitaban a los aspirantes a puestos de elección popular a publicar sus declaraciones de impuestos, potenciales conflictos de intereses e información sobre su patrimonio. ${ }^{31}$ De hecho, en los meses previos e inmediatamente posteriores a los hechos de Ayotzinapa y a la Casa Blanca, se había formado un grupo de académicos y activistas para discutir con legisladores, funcionarios y empresarios una serie de propuestas de reforma anticorrupción. Ese grupo incluyó académicos como Mauricio Merino y Enrique Cárdenas, y activistas y figuras públicas como Eduardo Bohórquez, Juan Pardinas, Haydeé Pérez, Max Káiser,

30 Según datos de Parametría, tras el escándalo de la "Casa Blanca" y durante la investigación de la SFP, la aprobación del presidente Peña Nieto pasó de $51 \%$ en octubre de 2014 a $39 \%$ en marzo de 2015. En cambio, el nivel de desaprobación pasó de $49 \%$ a $58 \%$ en el mismo periodo (véase: Parametría, “Tercer año de gobierno de EPN”, www.parametria.com.mx/ carta_parametrica.php?cp=4823 (consulta del 29 de enero de 2019).

31 "Cronología: Anticorrupción, la reforma que no termina de cuajar”, Expansión, 5 de julio de 2016. 
Edna Jaime y Lourdes Morales, entre varios otros. De estas discusiones surgieron muchas de las ideas que después se integrarían a la reforma constitucional y luego a la legislación secundaria. ${ }^{32}$

Por otro lado, la oposición aprovechó el momento para dar a conocer distintas iniciativas de ley. Por ejemplo, el Partido Acción Nacional (PAN) retomó una iniciativa de 2013 que finalmente sería el germen de la reforma anticorrupción de 2015. A ésta se añadieron poco después cuatro proyectos de los grupos parlamentarios del Partido del Trabajo y del Partido de la Revolución Democrática (PRD) en la Cámara de Diputados. ${ }^{33}$

Segundo, el descontento social tuvo eco en múltiples diagnósticos técnicos y académicos que enfatizaban la existencia y la gravedad del problema de corrupción endémica en el país. ${ }^{34}$ A esto se sumó el trabajo de investigación de periodistas y el apremio de la red de académicos, activistas y organizaciones de la sociedad civil que, como ya decía, habían participado en la reforma de 2014 (en materia de transparencia), en la Alianza por el Gobierno Abierto, en la campaña \#3de3 y en las mesas de discusión con legisladores y funcionarios. ${ }^{35}$ Este frente ciudadano fue capaz de ejercer presión públicamente. Más aún, tras la promulgación de la reforma constitucional de mayo de 2015 , ese grupo de activistas y organizaciones recolectó más de 630000 firmas de ciudadanos para respaldar una iniciativa de ley que popularmente se co-

${ }^{32}$ Hay varios recuentos de este periodo de voz de sus protagonistas en el libro de Alejandra Lajous, op. cit., 2019.

33 "Decreto por el que se reforman, adicionan y derogan diversas disposiciones de la Constitución Política de los Estados Unidos Mexicanos, en materia de combate a la corrupción", Diario Oficial de la Federación, 27 de mayo de 2015.

${ }^{34}$ Véase, por ejemplo, María Amparo Casar, México: Anatomía de la corrupción, México, IMCO, 2015.

${ }^{35}$ Véase Pedro Gerson y Fernando Nieto, Opening government? The case of Mexico in the Open Government Partnership, 2016, www.globalintegrity. org/wp-content/uploads/2018/12/Mexico-final.pdf (consulta del 1 de febrero de 2019). 
noció como la "Ley 3de3". ${ }^{36}$ Ésta recuperaba el espíritu de la campaña \#3de3 y lo plasmaba en una propuesta de Ley de Responsabilidades Administrativas que se construía sobre cinco pilares: un nuevo servidor público, eficacia ante la corrupción, sociedad responsable, nueva cultura de la denuncia y sanciones útiles. Tras ser acogida por senadores del PAN y PRD, la propuesta ciudadana de la Ley 3 de3 influiría directamente en la arquitectura del SNA.

Un tercer factor que explica la adopción de la reforma de 2015 es el precedente de la reforma en materia de transparencia. En particular, al menos dos elementos son relevantes. Primero, esta reforma había sido exitosa porque, entre otros factores, se hizo del apoyo de expertos, analistas y activistas de la sociedad civil. Segundo, la reforma se había decantado, tras la promulgación de la Ley General de Transparencia, en la creación de un Sistema Nacional (de Transparencia, SNT). En otras palabras, el precedente de la reforma de 2014 estableció parámetros legítimos para la negociación de la reforma anticorrupción y también ofreció una solución alternativa a la creación de una comisión nacional. Antes de la reforma en materia de transparencia, la discusión parlamentaria se había centrado en la creación de una nueva burocracia, de un "zar anticorrupción" o de una comisión de integridad (con distintas características y atribuciones, dependiendo del proponente). ${ }^{37}$ La idea de crear un Sistema Nacional Anticorrupción tiene, al menos en parte, su origen en el SNT, en las propuestas de la red de activistas y académicos (especialmente los congregados por la Red por la Rendición de Cuentas) ${ }^{38}$ y en la propuesta de reforma constitucional de 2013 del grupo parlamentario del PAN. Esta última ya había planteado

36 Iniciativa ciudadana contra la corrupción, Ley 3 de 3, www.le y3de3.mx

37 Eduardo Bohórquez, "Por un Sistema Nacional Anticorrupción 2.0", Letras Libres, 2 de enero de 2018, www.letraslibres.com/mexico/re vista/por-un-sistema-nacional-anticorrupcion-20-0 (consulta del 7 de febrero de 2019).

38 Alejandra Lajous, op. cit., pp.127-128. 
modificar el artículo 113 de la Constitución para hacer de la política anticorrupción una obligación concurrente de los tres órdenes de gobierno; creaba un mecanismo de coordinación entre diferentes autoridades de investigación y fiscalización, y establecía una instancia formal de discusión con presencia de consejeros ciudadanos honoríficos. ${ }^{39}$ Así, para principios de 2015 había ya precedente de una solución organizacional y normativa basada en la coordinación de las diferentes agencias y órganos del Estado con atribuciones relacionadas con el combate a la corrupción.

La conjunción de estos factores (un entorno político adverso a la administración peñista, la identificación de un problema de corrupción e impunidad extendidas y el precedente de la reforma de 2014) terminó por generar condiciones propicias. En febrero de 2015 cuajó la idea de crear un sistema nacional como una instancia de coordinación entre las autoridades de todos los órdenes de gobierno, con competencia en la prevención y sanción de la corrupción, así como con atribuciones para la fiscalización y el control interno de las organizaciones gubernamentales. El 27 de mayo de ese año se aprobó la reforma a la Constitución y se inició la discusión del paquete de legislación secundaria en el Senado de la República, con la participación activa de la sociedad civil. ${ }^{40}$

\section{LA ARQUitectura del Sistema NaGional ANTicorrupción}

La Ley General del Sistema Nacional Anticorrupción (LGSNA) establece que el propósito del sistema es crear "principios, bases generales, políticas públicas y procedimientos para la coordinación entre las autoridades de todos los órdenes de gobierno en la prevención, detección y sanción de faltas ad-

39 USAID y Ethos, Sistema Nacional Anticorrupción y el nuevo régimen de responsabilidades de los servidores públicos, México, 2017, pp. 22-23, www. ethos.org.mx/es/ethos-publications/sna-y-el-nuevo-regimen-de-respon sabilidades-2 (consulta del 25 de enero de 2018).

${ }^{40}$ Ibid., p. 24. 
ministrativas y hechos de corrupción, así como en la fiscalización y control de recursos públicos". ${ }^{41}$

Según su diseño original, el sNA se sostiene en cuatro pilares: coordinación interorganizacional e intergubernamental, nuevo régimen de responsabilidades, información para el combate a la corrupción y participación ciudadana. El sistema es, ante todo, un mecanismo de coordinación interinstitucional que abarca los tres poderes (coordinación horizontal) en los tres ámbitos de gobierno (coordinación vertical o intergubernamental). El primer pilar, por tanto, son las instancias de coordinación dentro del sna. Segundo, la reforma de 2015 inauguró un nuevo régimen de responsabilidades de los servidores públicos y de particulares que se plasmó en la Ley General de Responsabilidades Administrativas. También se crearon nuevos tipos penales relacionados con actos de corrupción en el Título X del Código Penal Federal. El tercer pilar es la Plataforma Digital Nacional (PDN). Ésta será un instrumento informático que contendrá datos sobre la evolución patrimonial y las declaraciones fiscales y de intereses de los servidores públicos, datos de los servidores públicos que intervienen en contrataciones, los servidores públicos y particulares que han sido sancionados, datos sobre denuncias sobre faltas y hechos de corrupción, el Sistema de Información Pública de Contrataciones, así como los sistemas de comunicación del propio SNA y del Sistema Nacional de Fiscalización. ${ }^{42}$ Finalmente, el cuarto pilar del sNa es el Comité de Participación Ciudadana que cumple la función, por medio de su presidente, de liderar el sistema en su conjunto.

Aunque no tengo espacio aquí para hacer un análisis a profundidad de la arquitectura jurídica y organizacional del SNA, ni del nuevo régimen de responsabilidades, ${ }^{43}$ es impor-

${ }^{41}$ LGSNA, art. 6.

42 Ibid., art 49. También véase SFP, Análisis de modelo de la Plataforma Digital Nacional, 2018, http://sna.org.mx/wp-content/uploads/2018/06 /2.pdf (consulta del 23 de enero de 2019).

${ }^{43}$ Para mayor detalle, véase María Amparo Casar, México: Anatomía de la corrupción ( $2^{a}$. edición, corregida y aumentada), México, Mexicanos contra 
tante al menos señalar sus principales elementos. Para empezar, el sNA está compuesto de tres espacios de coordinación, cuyo fin es articular objetivos y políticas en todo el país. El primer espacio es el Comité Coordinador en el que participan la SFP, el INAI, la Auditoría Superior de la Federación, el Tribunal Federal de Justicia Administrativa, la Fiscalía Especializada de Combate a la Corrupción, el Consejo de la Judicatura Federal y un representante del Comité de Participación Ciudadana, que preside el Comité Coordinador. ${ }^{44}$ El Comité tiene como propósito dirigir el SNA, así como formular y evaluar las políticas de combate a la corrupción. Para asistirlo en sus funciones, el Comité cuenta con la Secretaría Ejecutiva del Sistema Nacional Anticorrupción (SESNA), que es el principal brazo ejecutivo del sistema. ${ }^{45}$

La LGSNA también formalizó el Sistema Nacional de Fiscalización. Este segundo espacio de coordinación agrupa a la Auditoría Superior de la Federación, la sFP, las entidades de fiscalización superiores de las entidades y las contralorías locales o dependencias encargadas del control interno a nivel local. El Sistema de Fiscalización es una instancia de coordinación interinstitucional con el propósito de adoptar acciones y mecanismos transversales para mejorar la fiscalización de las haciendas y el control interno de las administraciones públicas del país. ${ }^{46}$

Finalmente, el tercer espacio de coordinación es la red de sistemas locales anticorrupción. La reforma constitucional de 2015 ordenó a los congresos locales integrar sistemas locales anticorrupción con una estructura análoga al sNA

la Corrupción y la Impunidad, 2016, pp. 77-86; usAId y Ethos, op. cit., pp. 27-47; y Eber O. Betanzos, Reforma en materia de combate a la corrupción, México, FCE, 2017, pp. 29-56.

${ }^{44}$ LGSNA, art. 10.

45 Ibid., cap. IV.

46 Ibid., Título III. Nótese que la participación del INAI en el Comité Coordinador del SNA también garantiza, al menos en principio, la vinculación con el Sistema Nacional de Transparencia, Acceso a la Información y Protección de Datos Personales que encabeza el mismo InAI. 
-esto es, con un comité coordinador de las autoridades con atribuciones de combate y prevención de la corrupción, un consejo de participación ciudadana y un brazo ejecutor similar a la SESNA-. Estos sistemas están a cargo de formular y ejecutar las políticas locales anticorrupción. La LGSNA mandata que todos los sistemas locales deben adoptar las metodologías y directrices que emita centralmente el sNA. ${ }^{47}$

Además de las instancias de coordinación del sNA, la reforma de 2015 transformó el régimen de responsabilidades de los servidores públicos. Este nuevo régimen establece la obligatoriedad de presentar las declaraciones de intereses y patrimonial, y un nuevo protocolo de compras y contrataciones públicas. Además, estipula faltas administrativas graves y no graves de los servidores públicos, comprendiendo un amplio catálogo como cohecho, desvío de recursos públicos, peculado, ocultamiento del conflicto de intereses y abuso de funciones. El nuevo régimen no sólo abarca al sector público, sino que también incluye responsabilidades de los particulares y la posibilidad de sanciones económicas a empresas por su participación en actos de corrupción. Por último, la reforma al Código Penal Federal, afinó e introdujo nuevos tipos penales.

Otro elemento central para el SNA, como ya he mencionado, es la Plataforma Digital Nacional. Ésta agrupará datos que son instrumentales en el combate y prevención de la corrupción. La idea detrás de la PDN es crear no sólo un repositorio de datos, sino también mecanismos digitales de comunicación y análisis que faciliten las tareas de las diferentes autoridades, incluyendo la coordinación y evaluación de las políticas anticorrupción. La PDN agrupa diferentes sistemas digitales que, en su mayoría, ya existen en la administración pública federal, si bien, hasta la fecha, aún deben conectarse y hacerse interoperables. ${ }^{48}$

${ }^{47}$ LGSNA, art. 36.

48 Véase SFP, op. cit. 
Por último, el Comité de Participación Ciudadana es una de las innovaciones de la reforma de 2015 y consistió en establecer en el centro del SNA un cuerpo colegiado de ciudadanos seleccionados por una comisión independiente. La lógica de esta decisión proviene posiblemente de la recomendación de ciudadanizar el combate a la corrupción a partir de la inclusión de una instancia independiente de propuesta y de vigilancia de los trabajos del sistema. Pero también es posible que la inclusión del Comité de Participación Ciudadana respondiese al impulso de institucionalizar la participación de la sociedad civil, misma que, como mencioné antes, había ganado espacios importantes de influencia. En cualquier caso, el Comité de Participación Ciudadana (al igual que sus homólogos en los estados) tiene una función central de supervisión, propuesta interna y liderazgo del sistema. Esto incluye la posibilidad de proponer proyectos e iniciativas, así como indicadores y mecanismos de evaluación de la política anticorrupción. ${ }^{49}$

\section{Problemas DE imPLEMENTACión}

La promulgación de la reforma anticorrupción de 2015 y la creación del SNA fueron ampliamente celebradas por la administración peñista y la mayoría de las fuerzas políticas. También fueron aplaudidas por organizaciones de la sociedad civil, académicos y organismos internacionales. ${ }^{50}$ Empero, a unos meses de su inauguración, el sistema y la reforma se enfrentaron a importantes retos y limitaciones en su implementación que aún hoy no se han salvado del todo y que generan dudas sobre su viabilidad en el mediano y largo plazos. Es decir, las limitaciones del sNA surgen de un diseño y arranque

${ }^{49}$ LGSNA, cap. III.

${ }^{50}$ Organización de Cooperación y Desarrollo Económicos, OCDE, Integrity Review of Mexico: Taking a Stronger Stance Against Corruption, París, OECD Public Governance Reviews, París, OECD Publishing, https://doi.org//10.1 787/9789264273207-en, p. 16. 
imperfectos, y también se traducen en retos a futuro. Los principales retos pueden clasificarse siguiendo el modelo de implementación de Michael Hill y Peter Hupe en: a) características de la reforma y problemas de diseño, b) recursos, c) relaciones intergubernamentales y d) cumplimiento y respuesta de los receptores de la reforma. ${ }^{51}$

\section{a) Características de la reforma y problemas de diseño}

Uno de los principales problemas del sNA es la complejidad organizacional inherente en su diseño jurídico. La lógica de coordinación que subyace a todo el sistema tiene la ventaja potencial de incitar sinergias entre las diferentes autoridades con competencia en materia anticorrupción. Sin embargo, también tiene la gran desventaja de que su operatividad depende en gran medida de la colaboración de todas las partes, la coherencia en las acciones y en la concertación cultivada desde el Comité de Participación Ciudadana. Estas condiciones presentan retos importantes. Como apunta Eduardo Bohórquez:

[...] la coordinación al interior de los sistemas anticorrupción se puede dar de iure o de facto. México decidió seguir la primera vía $[\ldots]$. Al escoger esta vía, el Congreso mexicano [...] pretende que los tres poderes del Estado (el ejecutivo, el legislativo y el judicial) hagan su parte en la tarea anticorrupción, al tiempo que la sociedad, representada por los ciudadanos que ahora participan y presiden el SNA, le den dirección y sentido estratégico al sistema en su conjunto. ${ }^{52}$

Por ende, el diseño del SNA plantea retos o condiciones de éxito respecto a la efectividad del mecanismo de coordi-

${ }^{51}$ Michael Hill y Peter Hupe, Implementing public policy, Londres, Sage, 2002, pp. 120-136.

${ }^{52}$ E. Bohórquez, "Por un Sistema Nacional...", art. cit. 
nación que, hasta ahora, no han sido cumplidas a cabalidad. Primero, la colaboración de las diferentes autoridades ha sido intermitente cuando debería ser constante e integral. Esto se debió, entre otros factores, a la ausencia de la fiscalía especializada autónoma ${ }^{53}$ y a la falta de una estrategia unificada que permita articular y, sobre todo, establecer las responsabilidades específicas de cada parte del snA. Más aún, no es claro que éste, o alguna de sus partes, tenga herramientas suficientes (legales o de otro tipo) para compeler la coordinación hacia dentro del sistema. Esto es particularmente evidente en el caso del Comité de Participación Ciudadana que, hasta ahora, se ha encontrado en una posición de clara desventaja organizacional y de recursos frente al resto de las autoridades y dependencias. Además, en el caso específico del Comité de Participación Ciudadana, la arquitectura legal del snA da lugar a ambigüedades sobre el papel y el peso relativo que puede o debe tener este comité dentro del sna; sobre todo, respecto a la definición y evaluación de las políticas anticorrupción.

Segundo, otro problema de diseño del sNa es la coordinación que supone, con al menos otros tres sistemas nacionales: de transparencia, de fiscalización y de archivos. Este segundo reto se refiere a la capacidad de coordinación entre sistemas con múltiples actores y con lógicas de operación y coordinación que no necesariamente son equivalentes. En otras palabras, el diseño del sNA produce dos problemas de coordinación. Uno de ellos (de primer orden), como ya decía, consiste en lograr una actuación y estrategia coordinadas dentro del sistema. Del otro lado, hay un problema de coordinación (de segundo orden) en lograr que la estrategia y actividades del sistema anticorrupción sean coherentes con

53 Según el decreto de la reforma de 2015, el Senado debía nombrar un Fiscal Especializado de Combate a la Corrupción en julio de 2017. Sin embargo, el nombramiento del nuevo fiscal especializado no se concretó sino hasta dos años más tarde. 
las políticas nacionales de transparencia, fiscalización y archivística. Como lo explica Joel Salas:

El acceso a la información y la transparencia son transversales a cada una de las fases que integran el ciclo de control de la corrupción. [...] De ahí la importancia de clarificar la vinculación entre el SNT y el SNA. Ubicar los espacios legislativos, jurídicos e institucionales donde ambos se intersectan nos permitirá saber a quién corresponde qué y poner a prueba este nuevo diseño institucional en la práctica. ${ }^{54}$

\section{b) Recursos}

En lo que respecta a los recursos, al ser un mecanismo de coordinación, el sNA no cuenta con una partida presupuestaria específica para su funcionamiento. No obstante, en 2018, el Presupuesto de Egresos de la Federación asignó recursos mediante un anexo transversal para "la prevención, detección y sanción de faltas administrativas y hechos de corrupción, así como para la fiscalización y control de recursos públicos". En total se asignaron, entre recursos fiscales y propios, poco más de 10.4 mil millones de pesos a diferentes dependencias y unidades. De estos, la SESNA obtuvo más de 172 millones de pesos. ${ }^{55}$ Así, puede afirmarse que, al menos en un inicio, hubo recursos para sentar algunas de las bases organizacionales del sistema.

Sin embargo, esta realidad cambió drásticamente al año siguiente, tras la elección de López Obrador. En el Proyecto de Presupuesto de Egresos de la Federación 2019 que la nueva administración entregó a la Cámara de Diputados, se omitió el sNa. Según un análisis del Presupuesto 2019 de Marco

${ }^{54}$ Joel Salas, "Prólogo", en El Sistema Nacional Anticorrupción y sus vínculos con el Sistema Nacional de Transparencia, México, UsAID, 2018, p. 3.

${ }^{55}$ Presupuesto de Egresos de la Federación (Anexo 20), Diario Oficial de la Federación, 29 de noviembre de 2017. 
Fernández, las autoridades que participan en el SNA vieron recortes nominales de entre $0.5 \%$ y $27.2 \%$ respecto al año anterior. El mayor recorte se dio en la SFP. En términos funcionales, el Presupuesto 2019 también recortó la mayoría de los programas anticorrupción del sexenio peñista, incluyendo el programa para la "promoción de la cultura de la integridad y el aprecio por la rendición de cuentas" y el programa para la "fiscalización a la gestión pública" ${ }^{56}$ Estos cambios implican que, apenas a unos meses de su lanzamiento, el SNA se enfrentó a una caída muy significativa en los recursos disponibles.

Otros dos retos relacionados con los recursos disponibles son la creación y consolidación de la PDN y la adquisición de recursos humanos. En lo que respecta a la PDN, tal y como se conceptualizó en la LGSNA, es un sistema muy ambicioso técnicamente que, empero, no tiene un propósito claro. Más aún, la construcción de la PDN se comenzó sin tener una política nacional anticorrupción, lo que implica que se ha invertido en la construcción de una herramienta sin tener claridad sobre su utilidad a la luz de una estrategia explícita. En lo concerniente a la adquisición de recursos humanos, la SESNA se constituyó con lentitud y aún hoy se encuentra en proceso de consolidación.

\section{c) Relaciones intergubernamentales}

Otro de los retos que ha enfrentado el sna es la configuración de los sistemas locales anticorrupción. Este reto de implementación puede, a su vez, dividirse en cuatro partes. Primero, las entidades deben adecuar sus marcos normativos incluyendo reformas a la constitución local para lograr la armonización

${ }^{56}$ Cit. en Nayeli Roldán, "Los siete organismos del Sistema Nacional Anticorrupción tendrán recorte para 2019”, Animal Político, 20 de diciembre de 2018, www.animalpolitico.com/2018/12/sistema-anticorrupcionrecorte-2019 (consulta del 8 de febrero de 2019). 
con el sNA y con la legislación general. Segundo, las entidades, al igual que en el caso del SNA, deben asignar recursos presupuestales y administrativos para el establecimiento y consolidación de los sistemas locales. Tercero, existe el supuesto de que los sistemas locales pueden hacer uso de capacidades establecidas en la sociedad civil local para integrar los comités de participación ciudadana. Finalmente, se supone que la organización en red del sNA permite que la diversidad y las particularidades de cada estado puedan acomodarse en diversas políticas anticorrupción y que, además, existe una comunicación y coordinación eficaz entre el sistema nacional y los sistemas locales.

Hasta ahora, no obstante, la implementación de los sistemas locales y el cumplimiento de las condiciones anteriores ha sido, en el mejor de los casos, incompleto. A pesar de que no existe un estudio sistemático que revise todos estos aspectos, sí hay evidencia de un desarrollo desigual con rezagos muy significativos. Por ejemplo, ante la falta de una política nacional que establezca directrices generales para todo el país, aún no ha sido posible desarrollar muchas de las políticas locales. Asimismo, la existencia de capacidades en las sociedades civiles locales ha demostrado ser un supuesto aventurado en algunos casos. Además, según el Semáforo Anticorrupción, hasta enero de 2019, la mayoría de las entidades tenía un nivel de implementación de reforma constitucional (local) satisfactoria, ocho entidades tenían una reforma de nivel regular y otras dos una reforma deficiente o que aún era una iniciativa en el congreso local. Respecto a la implementación de las leyes de los sistemas locales, 21 entidades habían alcanzado un nivel satisfactorio, ocho un nivel regular y tres no tenían ley o aún eran sólo una iniciativa. 57

57 Semáforo Anticorrupción, Monitoreo de los sistemas locales anticorrupción, www.semaforoanticorrupcion.mx 


\section{d) Cumplimiento y los receptores de la reforma}

Un último reto se refiere al cumplimiento de las directrices de la reforma. En este sentido, se han incumplido plazos importantes y, aún hoy, no se ha logrado integrar la totalidad del sistema. Tal parece que esta omisión se debe, al menos en parte, a plazos compulsivos establecidos en la reforma que no tomaron en cuentan las complejidades de implementación de un sistema tan ambicioso. Sumado a esto, el Senado de la República no nombró un fiscal especializado en el plazo obligatorio y fue omiso en el nombramiento de 18 magistrados del Tribunal de Justicia Administrativa.

Por otra parte, la SPF y el Tribunal Federal no han logrado modificar las rutinas de denuncia e investigación de responsabilidades de los servidores públicos. De hecho, el régimen transitorio de la reforma de 2015 no será superado sino hasta 2022, cuando prescribirán las responsabilidades de lo ocurrido bajo el régimen previo a la reforma. Además, persisten incongruencias en la Ley General de Responsabilidades Administrativas y falta de criterios para interpretar el nuevo régimen de responsabilidades.

\section{UN FUTURO INCIERTO}

Hacia finales de la administración peñista, la implementación del SNA aún se encontraba en una fase muy incipiente, con varios huecos y muchas limitaciones. Su diseño implica una coordinación interorganizacional compleja y difícil de lograr. A este problema, se añaden la falta de voluntad para integrar el sistema en su totalidad y comprometer recursos en su operación y consolidación, la ambigüedad respecto al papel del Comité de Participación Ciudadana y la ausencia de una política anticorrupción explícita. Más aún, en los últimos días de la administración de Peña Nieto no era claro que la continuidad del SNA estuviese garantizada. Esto no sólo se debió a la abierta hostilidad de la nueva administración hacia 
el sistema, sino a la débil coalición política, administrativa y ciudadana a favor del snA. Una de las sus principales debilidades es, pues, la ausencia de apoyos dentro y fuera, así como de la falta de credibilidad frente a los ciudadanos. Todos estos factores favorecen una visión pesimista sobre la estrategia y el legado anticorrupción del sexenio peñista.

Dicho legado, haciendo un balance, ha pecado de tres problemas que explican su debilidad intrínseca y permiten avizorar sus retos inmediatos tras el cambio de gobierno en 2018. Primero, la constitución del SNA y, de manera más general, los esfuerzos de combate a la corrupción durante el sexenio de Peña Nieto fueron marcadamente reactivos y condicionados. De hecho, puede argumentarse que el sNA en sí mismo es una respuesta tardía a un problema público que había sido plenamente identificado décadas atrás. ${ }^{58}$ La corrupción durante el peñismo fue un problema tratado principalmente de manera remedial y los remedios fueron, en su mayoría, poco creíbles. La reforma de 2015, por ejemplo, respondió al desbordamiento del descontento social y a la debilidad de la administración, misma que se vio en la necesidad de ceder en la negociación en el Senado ante la presión de la sociedad civil y la oposición. Además, como lo demuestran los datos, una vez que se promulgó la reforma, no se logró revertir la imagen negativa de la administración peñista, ni se logró convencer a los ciudadanos de la utilidad del SNA. ${ }^{59}$

Segundo, el resultado final (el SNA) fue incompleto tanto para hacer frente al problema público, como para galvanizar los recursos e instrumentos a que la ley lo obliga. A pesar de una inversión presupuestal importante en los primeros meses, los recursos en general se han mantenido precarios. Además, la falta de la Plataforma Digital Nacional y de la política nacional anticorrupción (aún hoy ausente), así como el retraso en la creación de instrumentos concretos (por ej., los formatos

58 Véase Dussauge, art. cit. y Valverde, art. cit.

${ }^{59}$ Ver supra, notas 8, 27 y 31. 
nacionales de declaración patrimonial y de intereses) han rezagado y entorpecido la operación de todo el sistema. Este retraso implicó que el SNA nunca terminara de consolidarse y que llegara con graves deficiencias operativas al sexenio de López Obrador.

Tercero, no es claro que exista -si acaso la hubo en algún momento- una voluntad decidida de consolidar y mejorar la estrategia anticorrupción. En este sentido, el legado anticorrupción no fue sólo reactivo e incompleto, sino también limitado. Es aún prematuro evaluar si esta limitación se extiende al impacto potencial del sistema (por ej., a los resultados de política pública), pero ciertamente se puede concluir que el sNA aún no ha logrado resolver el problema central de la reforma anticorrupción. Siguiendo a Anna Persson, Bo Rothstein y Jan Teorell, tal dificultad radica en que, para que el combate a la corrupción sea exitoso, es necesaria la voluntad política que haga efectivo el cambio institucional y, sobre todo, ejerza sanciones efectivas. En otras palabras, a menos de que se logre articular una coalición anticorrupción suficientemente poderosa y duradera, el liderazgo políticoadministrativo en países con corrupción endémica tiene pocos incentivos para cumplir con la reforma anticorrupción. ${ }^{60}$ En el caso del SNA aún no se ha logrado construir esa coalición, lo que impone un obstáculo mayor a la continuidad de la reforma (y a su efectividad).

Como he tratado de mostrar en las páginas anteriores, estos defectos pueden rastrearse en la configuración del Sistema Nacional Anticorrupción y en su incipiente trayectoria de implementación. También, es posible que estos defectos sean su principal debilidad ante la nueva realidad política del país. Durante la campaña electoral de 2018 y ciertamente tras la victoria de Andrés Manuel López Obrador, la corrupción y la impunidad se convirtieron en temas centrales de la agen-

${ }^{60}$ Anna Persson, Bo Rothstein y Jan Teorell, "Why anticorruption reforms fail - Systemic corruption as a collective action problem", Governance, vol. 26, núm. 3 (2013), pp. 449-471. 
da pública. Sin embargo, hasta ahora, la centralidad del tema no se ha traducido en un apoyo decidido de la nueva administración hacia el SNA. Esto no quiere decir que la trayectoria de implementación del sistema se haya detenido; sin embargo, sí constituye una evidencia en favor de la hipótesis de que los fallos y limitaciones del sistema no le han permitido consolidarse en una estrategia anticorrupción de largo plazo. En ese sentido, el legado anticorrupción del sexenio de Peña Nieto se mantiene en entredicho. Quizá, como decía al principio, el SNA esté condenado a no cumplir las expectativas originales. No obstante, hasta el momento, sigue siendo la apuesta más comprehensiva de combate y prevención de la corrupción en México. Los próximos años dirán si el legado del sexenio peñista será reivindicado con un sNA renovado y funcional o, en cambio, terminará en el olvido como otro esfuerzo malogrado por atajar uno de los problemas más apremiantes del país.

\section{REFERENCIAS BIBLIOGRÁFICAS}

Acuerdo A/011/14 por el que se crea la Fiscalía Especializada en Materia de Delitos Relacionados con Hechos de Corrupción y se establecen sus atribuciones, Diario Oficial de la Federación, 12 de marzo de 2014.

Ángel, Arturo, Duarte, el priista perfecto, México, Grijalbo, 2017.

Arellano Gault, D. y J. F. Hernández Galicia, “¿Control interno o lucha contra la corrupción? Hacia un verdadero control interno en la administración pública federal mexicana", en María del Carmen Pardo y Guillermo M. Cejudo (eds.), Trayectorias de reformas administrativas en México: Legados y conexiones, México, El Colegio de México, 2016, pp.149-184.

Ávila, Guillermo, "Citizen participation in legislative power: An argument to strengthen representation", en Eduardo Bohórquez (coord.), Open parliaments: Results and expectations, México, Transparencia Mexicana, 2015, pp. 57-70. 
Barrientos, F. y Daniel AÑorve, "México 2013: Acuerdos, reformas y descontento", Revista de Ciencia Política, vol. 34, núm. 1 (2014), pp. 221-247.

Betanzos, Eber O., Reforma en materia de combate a la corrupción, México, FCE, 2017.

Bohórquez, Eduardo, "Por un Sistema Nacional Anticorrupción 2.0", Letras Libres, 2 de enero de 2018, www.letraslibres.com/ mexico/revista/por-un-sistema-nacional-anticorrupcion-20-0 (consulta del 7 de febrero de 2019).

Casar, María Amparo, México: Anatomía de la corrupción, México, IMCO, 2015.

Casar, María Amparo, México: Anatomía de la corrupción (2a ${ }^{\text {. edi- }}$ ción, corregida y aumentada), México, Mexicanos contra la Corrupción y la Impunidad, 2016.

Castillo, Gustavo, "La maestra, presa, es acusada de lavado de dinero", La Jornada, 27 de febrero de 2013.

Cejudo, Guillermo, "Explaining change in the Mexican public sector: the limits of New Public Management", International Review of Administrative Sciences, vol. 74, núm. 1, 2008, pp. 111-127.

Cejudo, G., S. López Ayllón y A. Ríos Cázares, la política de transparencia en México. Instituciones, logros y desafíos, México, CIDE, 2012.

Corona, Jessica, "Las reacciones del presidente a lo ocurrido en su tercer año", Milenio, 1 de septiembre de 2015.

"Cronología: Anticorrupción, la reforma que no termina de cuajar”, Expansión, 5 de julio de 2016.

Decreto por el que se reforman, adicionan y derogan diversas disposiciones de la Ley Orgánica de la Administración Pública Federal, Diario Oficial de la Federación, 2 de enero de 2013.

Decreto por el que se reforman y adicionan diversas disposiciones de la Constitución Política de los Estados Unidos Mexicanos, en materia de transparencia, Diario Oficial de la Federación, 7 de febrero de 2014.

Decreto por el que se reforman, adicionan y derogan diversas disposiciones de la Constitución Política de los Estados Unidos Mexicanos, en materia de combate a la corrupción, Diario Oficial de la Federación, 27 de mayo de 2015. 
Dussauge Laguna, Mauricio I., "Combate a la corrupción y rendición de cuentas: avances, limitaciones, pendientes y retrocesos", en José Luis Méndez (coord.), Los grandes problemas de México: XIII Políticas Públicas, México, El Colegio de México, 2010, pp. 207-252.

García, Carina, "López Obrador anticipa fracaso del Sistema Anticorrupción”, El Universal, 3 de octubre de 2016.

Gerson, P., y F. Nieto, Opening government? The case of Mexico in the Open Government Partnership, 2016, www.globalintegrity.org/wpcontent/uploads/2018/12/Mexico-final.pdf (consulta del 1 de febrero de 2019).

Hill, Michael y P. Hupe, Implementing public policy, Londres, Sage, 2002.

Kingdon, John W., Agendas, alternatives and public policies, Crawfordsville, Pearson, 2011.

"La Casa Blanca de Enrique Pena Nieto", Aristegui Noticias, 9 de noviembre de 2014.

Lajous, Alejandra (coord.), La sociedad civil vs. la corrupción, México, Grijalbo, 2019.

Ley General de Transparencia y Acceso a la Información, 2015.

"Los compromisos que Peña Nieto tendrá que cumplir", Animal Político, 2 de julio de 2012.

Merino, Mauricio, México: La batalla contra la corrupción, Washington D.C., Wilson Center, 2015.

Myers Gallardo A., y Rouzand Anaya D. L., "El Sistema Nacional Anticorrupción”, Estudios, vol. 14, núm. 119, (2016), pp. 135-159.

Organización para la Cooperación y el Desarrollo Económicos, OCDE, Integrity review of Mexico: Taking a stronger stance against corruption, París, OCDE, 2017.

Parametría, Encuesta nacional en vivienda, www.parametria.com. $\mathrm{mx} /$ carta_parametrica. php?cp=4897 (consulta del 14 de diciembre de 2018).

Parametría, Tercer Año de gobierno de EPN, www.parametria.com.mx/ carta_parametrica.php?cp=4823 (consulta del 29 de enero de 2019). 
Persson A., B. Rothstein, y J. Teorell, "Why anticorruption reforms fail-Systemic corruption as a collective action problem", Governance, vol. 26, núm. 3, (2013), pp. 449-471.

Presupuesto de Egresos de la Federación (Anexo 20), Diario Oficial de la Federación, 29 de noviembre de 2017.

Rodríguez Nieto, Sandra et al., "La explicación de la SFP sobre las casas no convence ni a los viejos amigos de Peña Nieto: PRD y PAN", Sin Embargo, 23 de agosto de 2015.

RoldÁn, Nayeli, “¿Cuánto dinero desvió Duarte? La Auditoría dice que 60 mil mdp, la PGR que sólo 223 millones”, Animal Político, 18 de abril de 2017.

RoldÁn, Nayeli, "Los siete organismos del Sistema Nacional Anticorrupción tendrán recorte para 2019”, Animal Político, 20 de diciembre de 2018, www.animalpolitico.com/2018/12/siste ma-an ticorrupcion-recorte-2019 (consulta del 8 de febrero de 2019). Secretaria de la Función Pública (SFP), Análisis de modelo de la Plataforma Digital Nacional, 2018, http:/ / sna.org.mx/wp-content/ uploads/2018/06/2.pdf (consulta del 23 de enero de 2019).

Senadores del Grupo Parlamentario del Partido de la Revolución Institucional y Senadores del Grupo Parlamentario del Partido Verde Ecologista de México, Iniciativa con proyecto de decreto que reforma y adiciona los artículos 22, 73, 79, 105, 107, 109, 113, 116 y 122 de la Constitución Política de los Estados Unidos Mexicanos que presentan las senadoras y los senadores integrantes del grupo parlamentario del Partido Revolucionario Institucional y del Partido Verde Ecologista de México del Senado de la República, http://www.senado.gob.mx/comisiones/ puntos_constitucionales/docs/Corrupcion/Iniciativa_PRI.pdf (consulta del 18 de enero de 2018).

USAID, El Sistema Nacional Anticorrupción y sus vínculos con el Sistema Nacional de Transparencia, México, usaid, 2018.

USAID y Ethos, Sistema Nacional Anticorrupción y el nuevo régimen de responsabilidades de los servidores públicos, 2017, www.ethos.org. $\mathrm{mx} / \mathrm{es} /$ ethos-publications/sna-y-el-nuevo-regimen-de-respon sabilidades-2 (consulta del 25 de enero de 2018).

VAlverde, Miguel Ángel, "Mecanismos institucionales contra la corrupción”, en Fernando Nieto y Ernesto Velasco (eds.), Una 
agenda para la administración pública. Reconocimiento a la trayectoria de María del Carmen Pardo, México, El Colegio de México, 2018, pp. 265-296.

World Justice Project, The World Justice Project: rule of law index 20172018, Washington, The World Justice Project, 2018. 
